\title{
Hydrolysis of Oligosaccharides Over Solid Acid Catalysts: A Review
}

\author{
Léa Vilcocq, ${ }^{[a]}$ Paula C. Castilho, ${ }^{*[a]}$ Florbela Carvalheiro, ${ }^{[b]}$ and Luís C. Duarte ${ }^{[b]}$
}

\begin{abstract}
Mild fractionation/pretreatment processes are becoming the most preferred choices for biomass processing within the biorefinery framework. To further explore their advantages, new developments are needed, especially to increase the extent of the hydrolysis of poly- and oligosaccharides. A possible way forward is the use of solid acid catalysts that may overcome many current drawbacks of other common methods. In this Review, the advantages and limitations of the use of heterogeneous catalysis for the main groups of solid acid catalysts (zeolites, resins, carbon materials, clays, silicas, and other oxides) and their relation to the hydrolysis of model soluble disaccharides and soluble poly- and oligosaccharides are presented and
\end{abstract}

discussed. Special attention is given to the hydrolysis of hemicelluloses and hemicellulose-derived saccharides into monosaccharides, the impact on process performance of potential catalyst poisons originating from biomass and biomass hydrolysates (e.g., proteins, mineral ions, etc.). The data clearly point out the need for studying hemicelluloses in natura rather than in model compound solutions that do not retain the relevant factors influencing process performance. Furthermore, the desirable traits that solid acid catalysts must possess for the efficient hemicellulose hydrolysis are also presented and discussed with regard to the design of new catalysts.

\section{Introduction}

Biochemical platform development is in a more advanced stage in comparison to other biorefinery platforms; nevertheless, many improvements are still needed to further advance this concept. ${ }^{[1]}$ Specifically, the development and improvement of biomass fractionation technology and optimizing the fractionation processes to make use of the whole plant are an absolute necessity. Special attention should be given to hemicellulose separation, recovery, and valorization. Usually, hemicellulose is partly hydrolyzed and solubilized during the lignocellulose fractionation. This process, carried out in mild conditions using water (e.g., autohydrolysis, steam explosion), or using dilute acids, such as sulfuric or phosphoric acids, ${ }^{[2]}$ leads to the separation of a solid fraction containing cellulose and lignin and an aqueous fraction containing ex-hemicellulose poly/oligosaccharides (as the borderline between oligosaccharides and polysaccharides cannot be drawn strictly, ${ }_{1}^{[3]}$ for the sake of simplicity, oligosaccharides and soluble polysaccharides will be referred to as poly/oligosaccharides) and acetic acid, as well as ex-lignin and ex-proteins compounds (Figure 1).

Hemicellulosic poly/oligosaccharides (e.g., xylo-oligosaccharides) have an important added value for pharmaceutical and

[a] Dr. L. Vilcocq, Prof. P. C. Castilho

Centro de Química da Madeira

Universidade da Madeira

Campus da Penteada, 9020-105 Funchal (Portugal)

E-mail: castilho@uma.pt

[b] Dr. F. Carvalheiro, Dr. L. C. Duarte

Unidade de Bioenergia

LNEG (Laboratório Nacional de Energia e Geologia)

Uniadade de Bioenergia, Ed. K2

Est. do Paço do Lumiar 22, 1649-038 Lisboa (Portugal) food industries and could represent an economic advantage for the biorefinery. ${ }^{[4]}$ Nevertheless, their market volume is not large in comparison to the monosaccharide-derived products. From monosaccharides, even from the pentoses, conversion into bioethanol or biohydrogen is promising ${ }^{[5]}$ as well as the production of important platform molecules such as polyols or other fermentative products, furfural, 5-hydroxymethylfurfural (HMF), and levulinic acids, which are products of dehydration during acid hydrothermal conversion. ${ }^{[6]}$

Therefore, the hydrolysis of hemicellulose-derived poly/oligosaccharides into monomeric sugars is crucial in an integrated biorefinery framework, but process options are restricted to the use of sulfuric acid as enzymatic processes are still underperforming. ${ }^{[2]}$ However, mineral acids can be advantageously replaced by solid acid catalysts: heterogeneous catalysts can easily be separated, recovered, and reused; they are safe and non-corrosive. That is why they are increasingly used in numerous biorefinery processes. ${ }^{[7]}$ The use of solid acids keeps the advantages of using acid hydrolysis, but improves selectivity towards the glycosidic bonds at the expense of the dehydration of monosaccharides and can lead to higher sugar yield. Moreover, the hydrolysis of soluble oligosaccharides over a solid acid catalyst can be performed in a dynamic flow reactor, allowing for a continuous process and thus decreasing production cost as Kim et al. ${ }^{[8]}$ suggested in the case of corn-fiber valorization. Thus, solid acid catalysts represent an opportunity to develop more efficient and greener hydrolysis processes for soluble poly/oligosaccharides.

Cellulose deconstruction using solid acid catalysts has been studied in detail during the last decade. This is already the topic of several reviews. ${ }^{[9]}$ Comparatively, the catalytic hydroly- 